\section{Application of Deep Learning to Biomedical Informatics}

Received: November 02, 2016; Accepted: November 03, 2016; Published: November 09, 2016

Deep learning has become a popular machine learning technology since the first fast learning algorithm for training a deep belief neural net in 2006 [1]. There are over 2,800 deep learning publications indexed by Scopus only in the year of 2015. The origin definition of deep learning usually means the multi-layer artificial neural nets. Now-a-days, the definition has been generalized that any combination of computation models that "are composed of multiple processing layers to learn representations of data with multiple levels of abstraction" is called a deep learning model [2]. Deep learning has been successfully applied to many fields, such as image recognition [3], speech recognition [4], and machine translation [5], and embedded into industrial systems, like AlphaGo developed by Google DeepMind. The success of deep learning has brought new insights into the medical domain where there are large quantities of data available. For example, there are a large number of genes across the whole human genome and whole-genome gene expression profiling is still very expensive in typical academic labs to by considering a large number of conditions, such as genetic perturbations [6]. Taking advantage of the availability of "big data" in bioinformatics, deep learning has been widely utilized in gene expression regulation [7], protein structure prediction [8], drug discovery [9] and so forth.

Different from bioinformatics that applies informatics methods in biology and genetics, biomedical informatics is "the inter-disciplinary field that studies and pursues the effective uses of biomedical data, information, and knowledge for scientific inquiry, problem solving, and decision making, driven by efforts to improve human health" [10]. Electronic Health Records (EHR) is a typical kind of biomedical data that maintain information about an individual's health status and health care. Applications of deep learning to biomedical informatics research mainly focus on how to leverage EHR data for clinical decision support.

Medical images are one of the important resources stored in EHR. Following the traditional routine, researchers train deep learning models for feature representations and apply the pre-trained features to high-level tasks, such as classification, detection, and segmentation. Here are a few examples: for classification of tumor architecture, learning the features of histopathology tumor images with a deep neural net could improve the classification accuracy [11]; use of con

\section{Yanshan Wang \\ Department of Health Sciences Research, Mayo Clinic, Rochester, MN, USA}

Corresponding author: Yanshan Wang

\section{Wang.Yanshan@mayo.edu}

Research Fellow, Department of Health Sciences Research, Mayo Clinic, MN, USA.

Tel: $+1-507-293-1382$

Citation: Wang Y. Application of Deep Learning to Biomedical Informatics. Int J Appl Sci Res Rev. 2016, 3:5.

convolutional neural networks (CNNs) even on non-medical image could improve identification of different types of pathologies in chest x-ray images [12]; CNNs is also used to learn hierarchical representations of images for segmentation of tibial cartilage in low field knee MRI scans [13]; a unified deep learning framework is developed for feature representation and automatic prostate MR segmentation [14]. Those studies show that researchers have attempted to apply deep learning models to clinical radiology research to assist physicians.

However, many studies still just investigate how to use the existing deep learning models for their clinical research purpose. Though this is very common in applied science, leveraging the medical domain knowledge and incorporating that knowledge into model design are still in need of investigation.

Another issue related to the deep learning application in image analysis is that it is with difficulty to interpret the results for physicians. Many studies have tried to interpret the deeper learning results by developing a mimic model. The drawback is that many risk factors that are important for physicians are replaced with hyper-factors that have high correlations. For examples, in the study of predicting bone fracture for the elderly, age and sex are important risk factors. But if one trains a deep learning model, age and sex might be underweighted and cardiovascular factors might be overweighed in the final feature representations as the latter is highly correlated with the former and becomes main contribution in the final feature representations. 
Free-textual data are another important resource in EHR, including clinical notes, pathology notes, radiology reports, discharge summaries, etc. Those data contains massive valuable information provided by medical professionals. The problem is those data are not standardized, which makes it difficult for computers to process. Natural language processing (NLP) techniques are recently widely used in extracting information from clinical free-texts. There are lots of studies that apply deep learning to common NLP tasks, for example, using word embedding's for named entity recognition [15], or using long short term memory networks relation extraction [16]. These deep learning methods proposed for common NLP tasks could be effortless to be transferred to applications on clinical text. However, the reality is that there are only few studies consider using deep learning for clinical NLP tasks and these studies are mostly tested on shared-task corpus, such as i2b2 corpus [17] and SemEval corpus [18].
There are many potential future directions for applying deep learning to biomedical informatics. How to utilize both medial images and clinical diagnosis reports for designing deep learning models would be very interesting to physicians and health care providers. Due to the protected health information ( $\mathrm{PHI}$ ) provided by the Health Insurance Portability and Accountability Act (HIPAA), making clinical data public or shareable is a big obstacle for developing deep learning models. Due to the lack of public available clinical data, it obstructs researchers in computer science to tackle real clinical problems. Nevertheless, deep learning could learn feature representations and work embedding's and represents $\mathrm{PHI}$ in encoded vectors, which would facilitate researchers to share the clinical data. In addition, through collaborations with hospitals and health care agencies, researchers will find more opportunities to apply these techniques. 


\section{References}

1 Hinton GE, Osindero S, Teh YW (2006) A fast learning algorithm for deep belief nets. Neural Comput 18: 1527-1554.

2 LeCun Y, Bengio Y, Hinton G (2015) Deep learning. Nature 521: 436444.

3 Tompson JJ, Jain A, LeCun Y, Bregler C (2014) Joint training of a convolutional network and a graphical model for human pose estimation. In: Advances in Neural Information Processing Systems 27: 1799-1807.

4 Hinton G, Deng L, Yu D, Dahl G, Mohamedet AR, et al. (2012) Deep neural networks for acoustic modelling in speech recognition: The shared views of four research groups. IEEE Signal Process Mag 29: 82-97.

5 Cho K, Van Merriënboer B, Gulcehre C, Bahdanau D, Bougares F, et al. (2014) Learning phrase representations using RNN encoder-decoder for statistical machine translation. Association for Computational Linguistics pp: 1724-1734.

6 Chen Y, Li Y, Narayan R, Subramanian A, Xie X (2016) Gene expression inference with deep learning. Bioinformatics 32: 1832-1839.

7 Alipanahi B, Delong A, Weirauch MT, Frey BJ (2015) Predicting the sequence specificities of DNA-and RNA- binding proteins by deep learning. Nat Biotechnol 33: 831-838.

8 Baldi P, Brunak S, Frasconi P, Soda G, Pollastri G (1999) Exploiting the past and the future in protein secondary structure prediction. Bioinformatics 15: 937-946.

9 Ma J, Sheridan RP, Liaw A, Dahl GE, Svetnik V (2015) Deep neural nets as a method for quantitative structure-activity relationships. J Chem Inf Model 55: 263-274.

10 Shortliffe EH, Cimino JJ (2006) Biomedical Informatics. Springer.

11 Le Q V, Han J, Gray JW, Spellman PT, Borowsky A, et al. (2012) Learning invariant features of tumor signatures. In: $20129^{\text {th }}$ IEEE International Symposium on Biomedical Imaging (ISBI) pp: 302-305.

12 Bar Y, Diamant I, Wolf L, Greenspan H (2015) Deep learning with non-medical training used for chest pathology identification. In: SPIE Medical Imaging. Vol International Society for Optics and Photonics pp: 4140V-94140V.

13 Prasoon A, Petersen K, Igel C, Lauze F, Dam E, et al. (2013) Deep feature learning for knee cartilage segmentation using a triplanar convolutional neural network. Med Image Comput Comput Assist Interv 16: 246-253.

14 Liao S, Gao Y, Oto A, Shen D (2013) Representation learning: a unified deep learning framework for automatic prostate MR segmentation. Med Image Comput Comput Assist Interv 16: 254-261.

15 dos Santos C, Guimaraes V, Niterói RJ, de Janeiro R (2015) Boosting named entity recognition with neural character embeddings. In: Proceedings of NEWS 2015 the Fifth Named Entities Workshop p: 25.

16 Xu Y, Mou L, Li G, Chen Y, Peng H, et al. (2015) Classifying relations via long short term memory networks along shortest dependency path. In: Proceedings of Conference on Empirical Methods in Natural Language Processing.

17 Lv X, Guan Y, Yang J, Wu J (2016) Clinical relation extraction with deep learning. Int J Hybrid Inf Technol 9: 237-248.

18 Li P, Huang H (2016) Clinical information extraction via convolutional neural network. 